\title{
Gastric lipoma
}

\author{
Hassan Seddik $^{1}$, Aida Frej ${ }^{1}$, Fedoua Rouibaa ${ }^{2}$, Hassan En-Nouali ${ }^{3}$, Fatima-Zohra El Hamdi ${ }^{1}$, \\ Ahmed Benkirane ${ }^{1}$ \\ ${ }^{1}$ Department of Gastroenterology II, Military Teaching Hospital Mohammed V, Rabat, Morocco \\ ${ }^{2}$ Department of Gastroenterology I, Military Teaching Hospital Mohammed V, Rabat, Morocco \\ ${ }^{3}$ Department of Radiology, Military Teaching Hospital Mohammed V, Rabat, Morocco \\ Email: seddikhassan@yahoo.fr
}

Received 1 April 2012; revised 11 June 2012; accepted 11 July 2012

\begin{abstract}
Lipomas of the gastrointestinal tract are rare; gastric lipomas account for only $5 \%$ of all gastrointestinal lipomas. Although they are usually asymptomatic and detected incidentally, severe symptoms can occur with larger lesions, such as bleeding, abdominal pain or obstruction. Currently, accurate diagnosis of gastric lipoma can be reached with a combination of endoscopic and imaging diagnostic techniques, which constitutes a very useful precondition in choosing an appropriate management. We report a new case of a gastric lipoma diagnosed via abdominal CT scan and endoscopic ultrasound in a 63-year-old woman. Through this observation the authors discuss the contribution of morphological examinations in this disease whose diagnosis was made in the past on surgical specimen.
\end{abstract}

Keywords: Stomach; Lipoma

\section{INTRODUCTION}

Lipomas of the gastrointestinal tract are rare. The most common site is the colon followed by the small intestine. Gastric lipomas are extremely rare with an incidence of $5 \%$ of all the gastrointestinal lipomas [1]. These tumors are benign, slow growing up, composed of mature adipose tissue. In the past, accurate preoperative diagnosis was very difficult even for symptomatic gastric lipomas. Actual imaging technologies and endoscopic ultrasound have become new approaches in the management of gastric lipomas. The authors report a new case of gastric lipoma highlighting the value of morphological examinations in the diagnosis of this disease.

\section{CASE REPORT}

A 63-year-old female patient was referred to our hospital with a 3 month history of upper abdominal pain. She had no prior abdominal surgery or any major illness. On ad- mission, physical examination was normal with no palpable mass. Upper gastrointestinal endoscopy revealed in gastric antrum a soft submucosal oval-shaped mass of about $2 \mathrm{~cm}$ in diameter (Figure 1). The “cushion” sign (mark on the mass after compression by a biopsy forceps) and "tenting" sign (easy retraction of the overlying mucosa with a biopsy forceps) were positive. Multiple gastric mucosa biopsies showed helicobacter pylori $(\mathrm{Hp})$ gastritis. An Endoscopic ultrasound (EUS) was indicated to confirm the submucosal nature of this mass and to clarify its disposition in relation to the different layers of the gastric wall. EUS showed a hyperechogenic lesion and homogeneous oval mass with regular contours, developed in the submucosa. Abdominal CT scan showed on the gastric antrum a homogeneous well-defined oval mass around $1.73 \times 1.4 \mathrm{~cm}$ in diameter with negative densitometry values (-60 Hounsfield units) that corresponds to fatty tissue (Figure 2). The diagnosis of gastric lipoma was suggested. A simple follow-up was decided and the regression of the upper abdominal pain was obtained with the treatment of $\mathrm{Hp}$ infection.

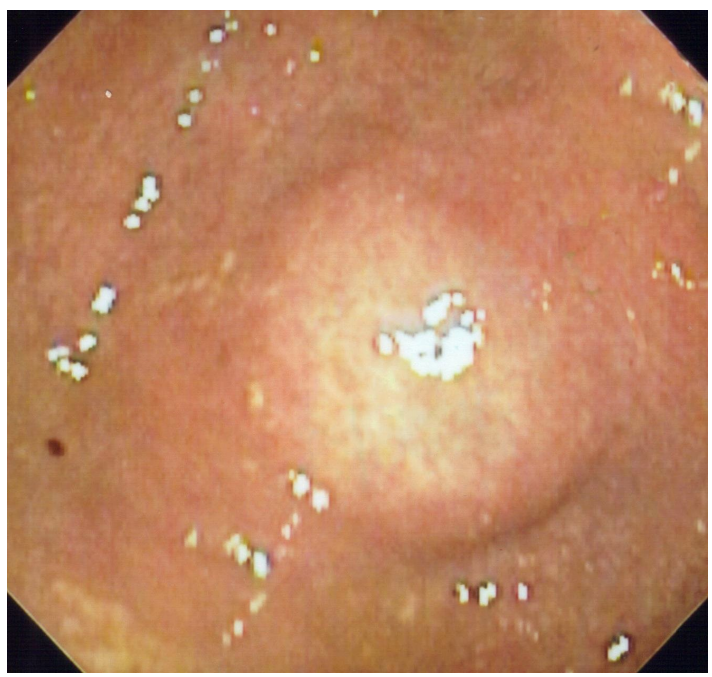

Figure 1. Endoscopic view showing a submucosal mass in gastric antrum. 


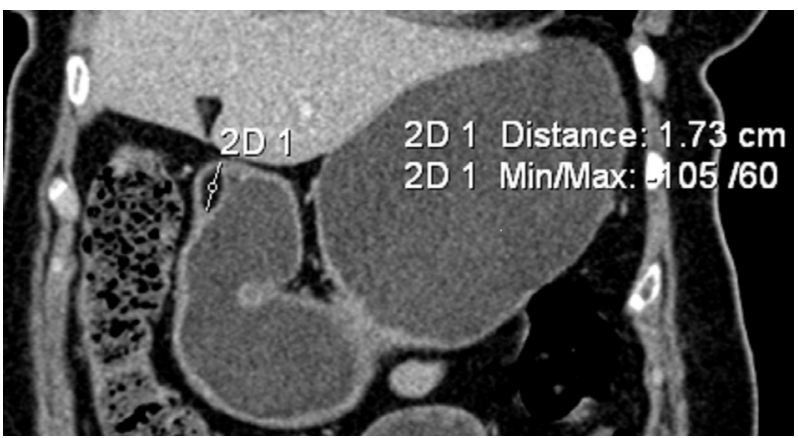

Figure 2. CT scan showing a mass with fat density, well demarcated in the wall of the gastric antrum.

\section{DISCUSSION}

Gastric lipomas are extremely rare with an incidence of $5 \%$ of all the gastrointestinal lipomas and have an incidence of $3 \%$ of all benign tumours of the stomach [1]. They are usually submucosal localized to the antrum and composed of mature adipose tissue [2]. Although they are usually asymptomatic and detected incidentally, severe symptoms can occur with larger lesions, such as bleeding, abdominal pain or obstruction [3-5]. The appearance of abdominal disorders depends on the size and location of lipomas [2].

Bleeding is the most common symptom (50\% of cases) [5], occurring due to ulceration of the mucosa. In the past, diagnosis of gastric lipoma was generally made after surgery. A treatment with mutilating procedures was sometimes performed for benign lipomas. Actual imaging technologies and endoscopic ultrasound allow an accurate diagnosis and have become new approaches in the management of gastric lipomas. Endoscopically, it appears as a submucosal mass with a soft consistency with or without central ulceration. The "cushion" sign (mark on the mass after compression by a biopsy forceps) and "tenting" sign (easy retraction of the overlying mucosa with a biopsy forceps) may guide diagnosis [1]. A clear-cut endoscopic differentiation between gastric lipomas and other submucosal neoplasm is not feasible. Usually biopsy does not reveal a diagnosis because it's a submucosal lesion with a normal mucosa overlying it. The endoscopic ultrasound is an excellent tool that can locate the lesion and which layer of the gastric wall is involved [6]. The typical form being a hyperechogenic mass with regular contours developed in the submucosal layer. CT scan is highly specific for diagnosis [7] by showing a well-defined mass of fat density. The specificity of both examinations can be reduced in case of tumor necrosis or ulceration, which can mask the lipomatous characteristics [7]. Gastric lipoma is a benign tumor without malignant potential; however, malignant lesions can be associated [8]. Indeed, the local chronic inflammation or repeated ulcerations of the gastric mucosa caused by large lipoma, could promote gastric cancer [8]. A complete preoperative exploration is necessary, so as not overlook synchronous gastric cancer. In our patient, preoperative evaluation did not find any morphologic and histological pathology in the gastric mucosa. The management of gastric lipomas is still controversial. Currently the different morphological examinations allow a precise preoperative diagnosis so an appropriate management of gastric lipomas. A simple follow-up or endoscopic resection is recommended in case of small lipomas. Surgical resection remains the treatment of choice for large and/or symptomatic lipomas [1].

Despite their rarity, gastric lipomas should be included in the differential diagnosis of masses of the stomach wall. In the past, the diagnosis was provided on a surgical resection. Currently, we have performant examinations that allow accurate preoperative diagnosis, which constitutes a very useful precondition in choosing an appropriate management.

\section{REFERENCES}

[1] Maderal, F., Hunter, F., Fuselier, G., Gonzales-Rogue, P. and Torres, O. (1984) Gastric lipomas-An update of clinical presentation, diagnosis and treatment. The American Journal of Gastroenterology, 79, 964-967.

[2] Singh, R. and Bawa, A.S. (2004) Lipoma of the stomach. Indian Journal of Surgery, 66, 177-179. doi:10.4321/S1130-01082010000600016

[3] Sadio, A., Peixoto, P., Castanheira, A., Cancela, E., Ministro, P., Casimiro, C., et al. (2010) Gastric lipoma-An unusual cause of upper gastrointestinal bleeding. Revista Española de Enfermedades Digestivas, 102, 398-400.

[4] Krasniqi, A.S., Hoxha, F.T., Bicaj, B.X., Hashani, S.I., Hasimja, S.M., Kelmendi, S.M., et al. (2008) Symptomatic subserosal gastric lipoma successfully treated with enucleation. World Journal of Gastroenterology, 14, 59305932. doi:10.3748/wjg.14.5930

[5] Vinces, F.Y., Ciacci, J., Sperling, D.C. and Epstein, S. (2005) Gastroduodenal intussusception secondary to a gastric lipoma. Canadian Journal of Gastroenterology, 19, 107-108.

[6] Yasuda, K., Cho, E., Nakajiama, M. and Kawai, K. (1990) Diagnosis of submucosal lesions of the upper gastrointestinal tract by endoscopic ultrasonography. Gastrointestinal Endoscopy, 36, 517-520. doi:10.1016/S0016-5107(90)71010-3

[7] Park, S.H., Han, J.K., Kim, T.K., Lee, J.W., Kim, S.H., Kim, Y.I., et al. (1999) Unusual gastric tumours: Radiologicpathologic correlation. Radiographics, 19, 14351446.

[8] Yamamoto, T., Imakiire, K., Hashiguchi, S., Matsumoto, J., Kadono, J., Hamada, N., Yoshioka, T. and Kitajima, S. (2004) A rare case of gastric lipoma with early gastric cancer. Internal Medicine Journal, 43, 1039-1041. doi:10.2169/internalmedicine.43.1039 